\title{
Hybrid Temperature Compensation Model of MEMS Gyroscope Based on Genetic Particle Swarm Optimization Variational Modal Decomposition and Improved Backpropagation
}

\author{
Jingru Wei, ${ }^{1}$ Zekai Zhang, ${ }^{1}$ Huiliang Cao, ${ }^{1,2}$ and Xiaomin Duan ${ }^{3 *}$ \\ ${ }^{1}$ School of Instrument and Electronics, North University of China, Taiyuan 030051, China \\ ${ }^{2}$ Science and Technology on Electronic Test \& Measurement Laboratory, North University of China, \\ Taiyuan 030051, China \\ ${ }^{3}$ School of Electronic Science and Engineering, University of Electronic Science and Technology of China, \\ Chengdu 610054, China
}

(Received April 20, 2021; accepted June 29, 2021)

Keywords: MEMS gyroscope, genetic particle swarm optimization (GPSO), genetic algorithm (GA), compensation

The output of a MEMS gyroscope is easily influenced by temperature, which has led to a bottleneck in the development of gyroscopes. Therefore, to eliminate the temperature error of gyroscopes, a parallel processing algorithm based on variational modal decomposition optimized by genetic particle swarm optimization variational modal decomposition (GPSO-VMD) and an improved backpropagation (BP) neural network is proposed in this paper. First, for the original output signal of a gyroscope, GPSO is adopted to search for the optimal parameters for VMD. Next, the optimal parameters $\left(k_{\text {best }}, \alpha_{\text {best }}\right)$ are applied to VMD to obtain intrinsic mode functions (IMFs). Then, according to the calculated result of multiscale permutation entropy (MPE), IMFs are divided into three categories: noise items, mixed items, and drift items. The three categories are treated separately: noise items are removed directly, mixed items are filtered, and for drift items, temperature errors are eliminated by using an improved BP neural network. The final signal is then obtained through reconstruction. Compared with the traditional optimization algorithm, GPSO has excellent global search ability and strong convergence. The BP neural network improved by the genetic algorithm (GA) overcomes the problem of easily falling into a local optimum, and excellent prediction performance is achieved. Experimental results demonstrate the feasibility of this proposed hybrid model in eliminating gyroscope temperature errors.

\section{Introduction}

With the rapid development of MEMS technology, research on inertial devices such as accelerometers and gyroscopes has become a hotspot. ${ }^{(1-5)}$ MEMS gyroscopes are widely used in aviation, aerospace, and other high-precision measurement and control fields due to their low cost, low power consumption, robustness, and excellent performance. ${ }^{(6,7)}$ Unfortunately, the ${ }^{*}$ Corresponding author: e-mail: dxm@uestc.edu.cn https://doi.org/10.18494/SAM.2021.3412 
performance of MEMS gyroscopes is limited by many factors, such as background noise, humidity drift, and temperature drift, which has greatly inhibited their development. ${ }^{(8-14)}$ In terms of temperature drift, scholars have performed numerous studies on improving the gyroscope structure and the subsequent output signal processing to reduce the temperature error, and achieved outstanding results.

The methods of gyroscope temperature compensation can be roughly divided into hardware compensation and software compensation. Hardware compensation mainly involves optimizing the structure and circuit of the gyroscope. Cui et al. studied a temperature compensation model based on a scale factor, and were able to eliminate the temperature error by compensating the driving amplitude operating point. ${ }^{(15)}$ In Ref. 16, by designing a high-linearity amplifier with constant transconductance, the circuit of a gyroscope was optimized to eliminate the temperature error. A new control method to make the transmission shaft of a MEMS gyroscope resonate, thereby achieving temperature compensation, was analyzed by Zheng et al. ${ }^{(17)}$ However, hardware compensation has the disadvantage of a long implementation cycle and high cost.

The second method is software compensation. Its core idea is to further process the gyroscope output signal by adopting software. The processing aspect includes noise reduction of the highfrequency part and temperature drift compensation of the low-frequency part, and the final processed output signal is obtained through reconstruction. Parallel processing and serial processing are two different processes. Serial processing involves denoising followed by compensation, but it causes some static drift and affects signal processing. This led to the development of the parallel processing method, which mainly processes the noise part and the drift part separately at the same time. Fei et al. adopted a robust adaptive control strategy based on a fuzzy compensator, and the effectiveness of the strategy was verified by numerical analysis. ${ }^{(18)}$ In Ref. 19, a radial basis function neural network based on the genetic algorithm (GA) and Kalman filter was adopted, which greatly improved the bias instability of a gyroscope.

An important step in the software compensation method of parallel processing is the multiscale decomposition of the signal. Common signal decomposition methods include local mean decomposition and empirical mode decomposition, but they are essentially recursive decomposition methods, which cannot completely eliminate modal aliasing, the end effect, and other problems. ${ }^{(20,21)}$ By contrast, the variational modal decomposition (VMD) algorithm has a good decomposition effect for nonstationary nonlinear signals and can suppress clutter interference and mode aliasing to a certain extent, avoiding the defects of nonrecursive decomposition algorithms. ${ }^{(22)}$ However, the parameters of the VMD algorithm are artificially selected, and improper parameter selection will have a major impact on experimental results. Wang et al. optimized the parameters of VMD by adopting particle swarm optimization (PSO), which effectively extracted the signal features. ${ }^{(23)}$ However, PSO tends to converge prematurely and fall into a local optimum when searching for an optimal solution, resulting in poor optimization. To improve the accuracy and speed of optimization, Ma et al. proposed an immune particle swarm optimization (IPSO) algorithm. ${ }^{(24)}$ In this paper, we propose the use of genetic particle swarm optimization (GPSO) to optimize VMD, and the best decomposition effect is achieved through iterative optimization. Another key step is to establish an appropriate temperature compensation model. Artificial neural network prediction is favored by many 
researchers due to its superiority in gyroscope temperature compensation, and it has incomparable advantages in dealing with nonlinear problems. When the temperature compensation model uses a neural network prediction method, it is necessary to set the test set and the training set. The training set is the pre-obtained reference temperature and the corresponding temperature drift, and the test set is the data to be processed. After the temperature compensation model is established, the model can predict the output value as compensation when the test set is input. By using a neural network model, Shiau et al. established a temperature calibration mechanism that improved the calculation problem caused by temperature drift. ${ }^{(25)} \mathrm{Xia}$ et al. established a temperature control system based on a backpropagation (BP) neural network that could control the temperature error within $0.2{ }^{\circ} \mathrm{C} .(26)$ However, it did not overcome the inherent shortcoming of BP neural networks of easily falling into a local optimum. Therefore, in this paper, we improve the initial weights of the BP neural network by using GA, which gives the BP neural network better nonlinear mapping capability and prediction accuracy.

On the whole, the hybrid model proposed in this paper is a parallel processing method. The VMD algorithm optimized by GPSO is used for signal decomposition to obtain a series of intrinsic mode functions (IMFs), and then the multiscale permutation entropy (MPE) is used to judge the complexity of the time series and divide the components into noise items, mixed items, and temperature drift items. ${ }^{(27)}$ The noise items are removed directly because the information they contain is useless, and the noise items are denoised by using a forward linear prediction (FLP) filtering algorithm. For the temperature drift items, the BP network optimized by GA is used to predict and realize temperature compensation. Finally, the processed items are reconstructed to obtain the final output signals of the gyroscope. Experiments show that this method has excellent performance for the signal processing of gyroscopes.

\section{Dual-Mass MEMS Gyroscope}

The sample gyroscope used in this paper is a dual-mass MEMS gyroscope with a tuning-fork structure, as shown in Fig. 1. The gyroscope has two modes: the driving mode and the sensing

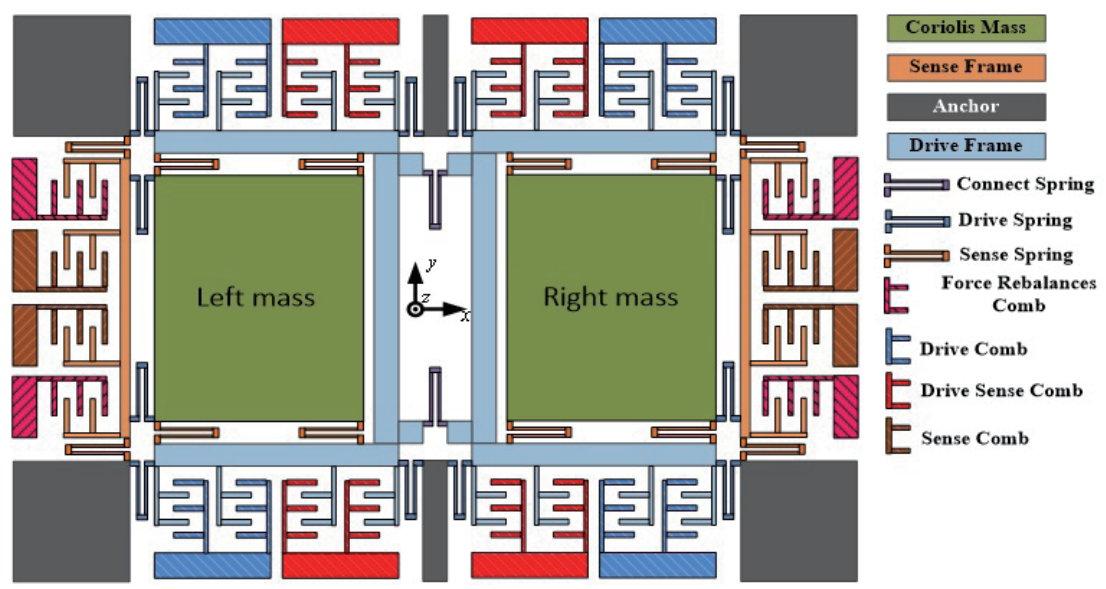

Fig. 1. (Color online) Schematic diagram of MEMS gyroscope structure. 
mode, and each mode is composed of three parts and contains frames, combs, and springs. The gyroscope also has two masses, with each mass a common part of the two modes, and the two modes are isolated from each other without coupling displacement. In addition, an automatic gain control (AGC) loop is adopted by the gyroscope to stabilize the vibration amplitude of the driving mode and improve the gyroscope's mechanical sensitivity. When static electricity acts on the driving mode, the two sensing masses are coupled by the $x$-axis warpage of the driving spring, and the left and right masses are connected and coupled by the connecting U-shaped spring.

The first four modes of the gyroscope structure are shown in Fig. 2. The left and right masses and the driving frame vibrate along the $x$-axis in the same direction. The actual working sensing mode is jointly constituted of the modes in Figs. 2(b) and 2(c), where Fig. 2(c) shows the thirdorder mode of the anti-phase mode, and Fig. 2(b) shows the second-order mode of perception of the in-phase mode. The sensing frame and the left and right masses move along the $y$-axis, and the directions of motion of the two masses are the same in Fig. 2(b) but opposite in Fig. 2(c). The fourth-order mode shown in Fig. 2(d) is the driving anti-phase mode, and this mode is the actual working driving mode, in which the left and right masses move in opposite directions along the

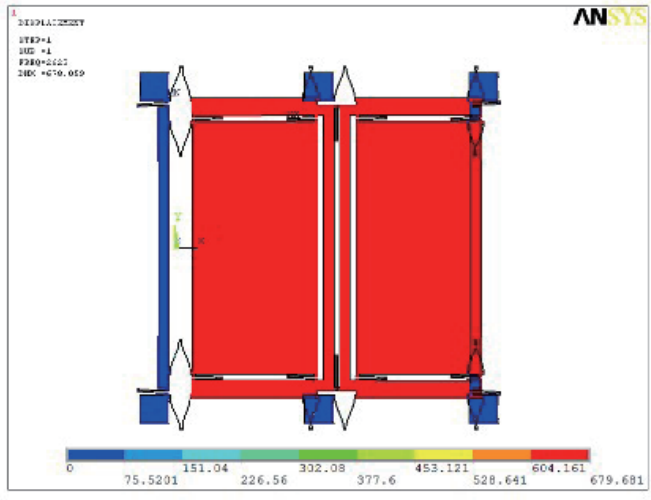

(a)

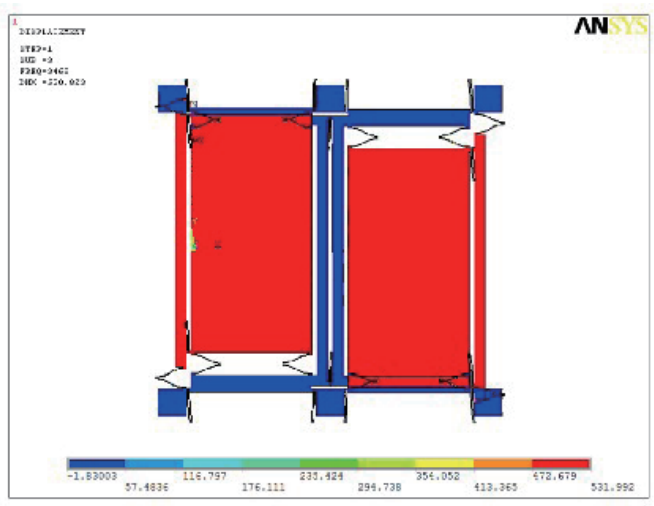

(c)

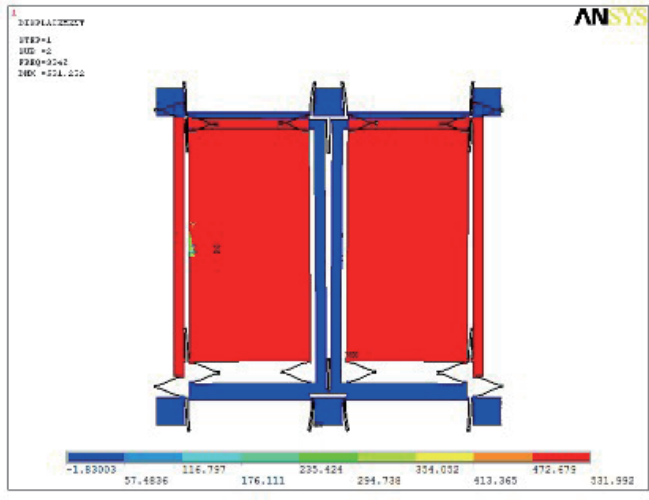

(b)

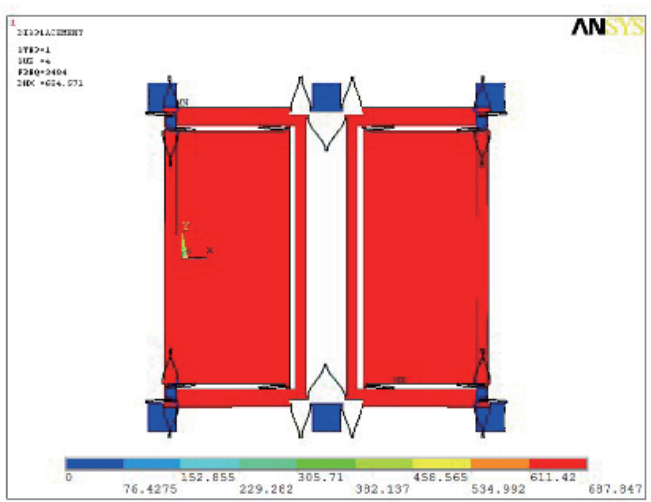

(d)

Fig. 2. (Color online) (a) Driving in-phase mode (first mode), (b) sensing in-phase mode (second mode), (c) sensing anti-phase mode (third mode), and (d) driving anti-phase mode (fourth mode). 
driving shaft. The electrostatic force can drive the driving mode, causing the two masses to vibrate in opposite directions along the $x$-axis. After the angular rate input $\Omega z$ around the $z$-axis is reached, the vibration mass generates the Coriolis force and transmits it to the sensing frame on the $y$-axis, and the monitoring circuit can monitor the whole process.

A schematic diagram of the MEMS gyroscope monitoring system is shown in Fig. 3. In the driving loop, the displacement of the drive frame $x(t)$ is detected by the drive sensing combs and extracted by a differential amplifier. To meet the phase requirement of the AC drive signal, which is $V_{d a c} \sin \left(\omega_{d} t\right)$, the phase of the signal is delayed by $90^{\circ}$. Then, full-wave rectification and low-pass filtering are used to extract the amplitude of the signal $V_{d a c} \sin \left(\omega_{d} t\right)$. The voltage $V_{d a c}$ in the comparator is then compared with the reference voltage $V_{r e f}$ A control signal generated by the integrator controller is used to drive the DC signal $V_{d c}$ to accumulate $V_{d a c} \sin \left(\omega_{d} t\right)$ to stimulate the driving mode. A differential detection amplifier is used to detect the left and right qualitysensing signals, and the output signal is processed by the secondary difference to generate the signal $V_{\text {stotal }}$. The sensing circuit is an open-loop circuit, which is similar to the driving circuit. The sensing signal $V_{\text {stotal }}$ is demodulated by $V_{d a c} \sin \left(\omega_{d} t\right)$ and filtered by a low-pass filter to obtain the motion signal $V_{\text {Oopen }}$. Finally, the sensing open-loop output signal is $V_{\text {Oopen }}{ }^{(28)}$

\section{Algorithms and Models}

\subsection{VMD}

VMD is a nonrecursive modal decomposition algorithm based on the Wiener filter. The signal can be decomposed into multiple IMF components with a certain bandwidth around the center frequency by adopting VMD. The VMD algorithm includes two parts: establishing the variational model and solving the variational model, ${ }^{(29)}$ and the details are as follows:

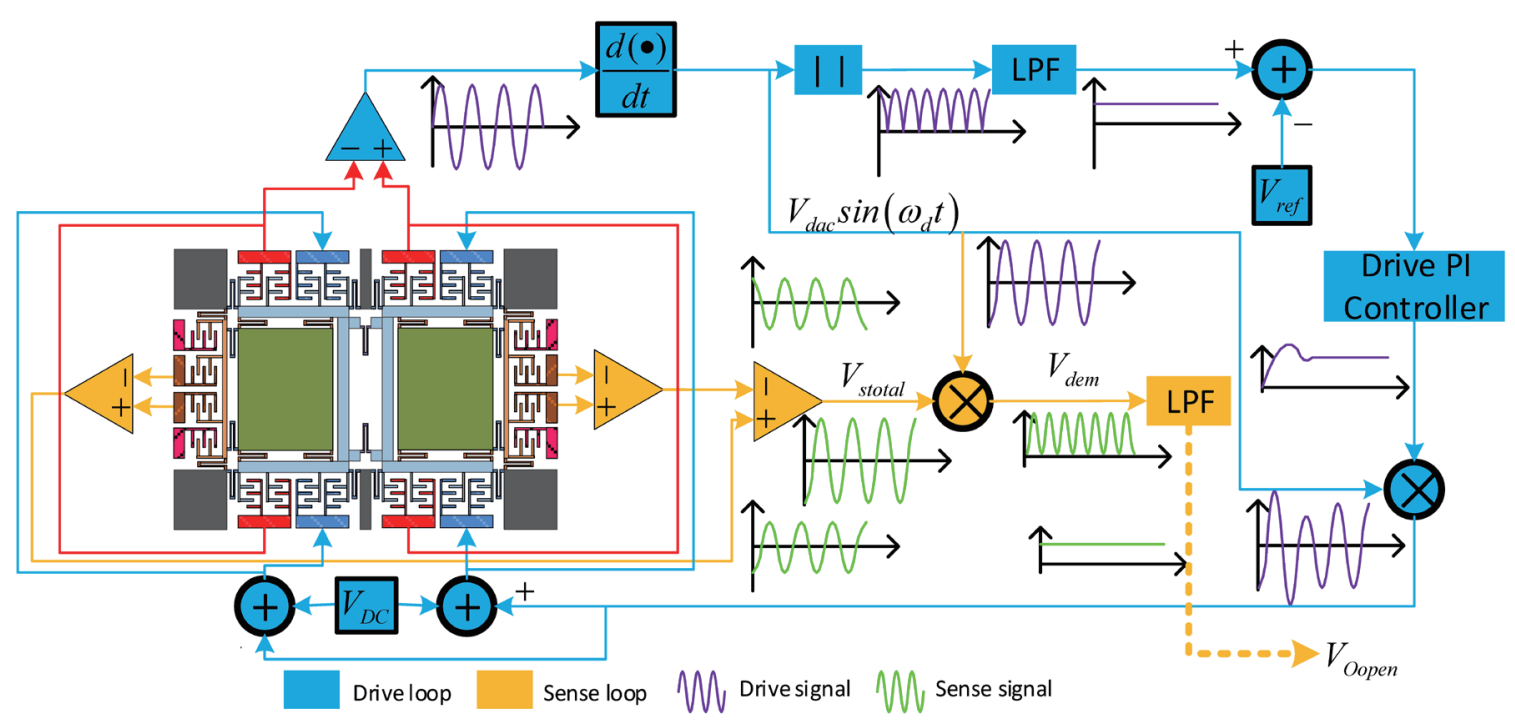

Fig. 3. (Color online) Schematic diagram of gyroscope monitoring system. 


\subsubsection{Establishment of variational model}

(1) Decompose each mode of the original signal $f$ to obtain $u_{k}(t)$, which has $k$ IMF components. Hilbert variations are performed on each mode to obtain its one-sided spectrum:

$$
\left[\delta(t)+\frac{j}{\pi t}\right] * u_{k}(t)
$$

where * represents the convolution operator.

(2) The center frequency of each $u_{k}(t)$ is estimated and its spectrum is modulated to the corresponding baseband, which is

$$
\left\{\left[\delta(t)+\frac{j}{\pi t}\right] * u_{k}(t)\right\} e^{-j \omega t}
$$

(3) A Gaussian smoothing index is used to estimate the bandwidth of each IMF component after the frequency shift, and the calculation process is transformed into a constrained variational problem:

$$
\min \left\{\sum_{k}\left\|\delta_{t}\left[\left(\delta(t)+\frac{j}{\pi t}\right) \times u_{k}\right] \times e^{-j \omega t}\right\|_{2}^{2}\right\} \text {, s.t. } \sum_{k=1}^{K} u_{k}=f,
$$

where s.t. is an abbreviation of "subject to" and indicates the constraints, and \|\| indicates the norm, which is a mapping from a linear space to a non-negative real number.

(4) The quadratic equilibrium constraint parameter $\alpha$ (also called the penalty factor) and the Lagrange multiplier $\lambda(t)$ are introduced to transform the variational problem into an unconstrained variational problem. The parameter $\alpha$ can ensure the reconstruction accuracy of the signal and avoid the interference of Gaussian noise, and $\lambda(t)$ can ensure the severity of constraints. Equation (4) is the Lagrange equation:

$$
\begin{aligned}
L\left(u_{k}, \omega_{k}, \lambda_{k}\right)= & \alpha \sum_{k}\left\|\partial_{t}\left[\left(\partial(t)+\frac{j}{\pi t}\right) \times u_{k}\right] \times e^{-j \omega t}\right\|_{2}^{2} \\
& +\left\|f(t)-\sum_{k=1}^{K} u_{k}(t)\right\|_{2}^{2}+<\lambda(t), f(t)-\sum_{k=1}^{K} u_{k}(t)>,
\end{aligned}
$$

where $\partial$ is the balancing parameter of the data-fidelity constraint. $<>$ is the inner product operator, where the inner product operation is to take the transpose of the first term and multiply it by the second term. 


\subsubsection{Solving the variational model}

The saddle points of the Lagrange expression are searched for by the alternative direction multiplier method, and the saddle points are the optimal solutions of Eq. (4). The algorithm is described as follows:

(5) Initialize $\left\{u_{k}^{l}\right\},\left\{\omega_{k}^{l}\right\},\left\{\lambda^{l}\right\}$, and the number of iterations $n$.

(6) Update $u_{k}$ and $\omega_{k}$ as follows:

$$
\begin{gathered}
\hat{u}_{k}^{n+1}(\omega)=\frac{\hat{f}(\omega)-\sum_{i \neq k} \widehat{u}_{i}(\omega)+\frac{\hat{\lambda}(\omega)}{2}}{1+2 \alpha\left(\omega-\omega_{k}\right)^{2}}, \\
\omega_{k}^{n+1}=\frac{\int_{0}^{\infty} \omega\left|\hat{u}_{k}(\omega)\right|^{2} d \omega}{\int_{0}^{\infty}\left|\hat{u}_{k}(\omega)\right|^{2} d \omega} .
\end{gathered}
$$

(7) Update $\lambda$ as follows:

$$
\hat{\lambda}^{n+1}(\omega)=\hat{\lambda}^{n}(\omega)+\tau\left[\hat{f}(\omega)-\sum_{k} \hat{u}_{k}^{n+1}(\omega)\right] .
$$

(8) The discriminant precision is $\varepsilon>0$. If the discriminant in Eq. (8) is satisfied, the algorithm stops. Otherwise, the calculation returns to step (6).

$$
\sum_{k} \frac{\left\|u_{k}^{n+1}-u_{k}^{n}\right\|_{2}^{2}}{\left\|u_{k}^{n}\right\|_{2}^{2}}<\varepsilon
$$

In Eqs. (5)-(7), $\widehat{u}_{i}(\omega), \widehat{u}_{k}^{n+1}(\omega), \hat{\lambda}(\omega)$, and $\hat{f}(\omega)$ denote the Fourier transforms of $u_{i}(t)$, $u_{k}^{n+1}(t), \lambda(t)$, and $f(t)$, respectively.

Although VMD has the property of nonrecursive synchronous decomposition, the selection of some parameters, such as the number of decomposition modes $k$ and the penalty factor $\alpha$, will have a significant influence on the decomposition results. A very large $k$ value will result in over-decomposition, and a very small $k$ value will result in underdecomposition. Similarly, the value of $\alpha$ will affect the bandwidth of the modal function; the larger the value of $\alpha$, the smaller the bandwidth.

\subsection{GPSO}

Because the choice of parameters in VMD has a significant influence on the decomposition result, the optimal parameters $\left(k_{\text {best }}, \alpha_{\text {best }}\right)$ in VMD are searched for by adopting the GPSO. 
GPSO combines the advantages of the strong global search ability of GA and the fast convergence of the PSO algorithm, which can greatly improve the optimization efficiency.

\subsubsection{GA}

GA was inspired by Darwin's theory of evolution. ${ }^{(30)}$ By simulating the genetic mechanism of nature and the theory of biological evolution, GA can find optimal solutions. A binary code is used to represent the genes of individuals and the details are described as follows:

1. Initialize the population and determine the population size, cross probability, mutation probability, and evolutionary termination rule.

2. Assess the fitness of individuals in the population.

3. Individuals with poor fitness are eliminated according to the mechanism of survival of the fittest, and the surviving individuals are randomly selected according to their fitness value. At the same time, the surviving individuals copy themselves to form a new population.

4. Surviving individuals replicate themselves to produce parents, and the parents carry out genetic operations to produce offspring. Genetic operations include crossover and mutation. Crossover is the exchange of genes between parents' chromosomes, and mutation is a sudden change in a gene's position. New individuals are created in these two ways.

5. The current population is propagated and new individuals are produced through selection, crossover, and mutation.

6. Evolution ceases if a preset termination condition is met. Otherwise, the algorithm returns to step 2.

In a complex space, GA adopts a probabilistic optimization method, which can adjust the search direction adaptively and obtain the optimized search space automatically. GA has the characteristics of parallelism, randomness, and robustness.

\subsubsection{PSO}

PSO is a global stochastic algorithm based on swarm intelligence that seeks the optimal solution through collaboration and information sharing between individuals in a complex space. After iterative optimization, a global optimal solution can be obtained, and the PSO algorithm is expressed as follows:

1. The particle swarm is randomly initialized in the velocity interval and search space to determine the initial position and velocity of the particle. The spatial dimension of the particle search is assumed to be $D$, the position of the $i$ th particle is denoted as $X_{i}=\left[x_{i 1}, x_{i 2}, x_{i 3}, \ldots, x_{i D}\right]$, and the velocity vector is denoted as $V_{i}=\left[v_{i 1}, v_{i 2}, v_{i 3}, \ldots, v_{i D}\right]$.

The individual extremum and global extremum can be obtained by calculating the fitness of particles. The individual extremum is the best position that each particle passes through, denoted by $P_{i b e s t}=\left[p_{i 1}, p_{i 2}, p_{i 3}, \ldots, p_{i D}\right]$, and the global extremum is the best position found in the population, which is $P_{g b e s t}=\left[p_{g 1}, p_{g 2}, p_{g 3}, \ldots, p_{g D}\right]$.

2. The velocity and position of the particles are updated. The velocity update formula of each particle in the iterative process is 


$$
V_{i}(k+1)=\omega V_{i}(k)+c_{1} r_{1}\left[p_{\text {ibest }}-X_{i}(k)\right]+c_{2} r_{2}\left[p_{\text {gbest }}-X_{i}(k)\right],
$$

where $k$ is the number of iterations, $c_{1}$ and $c_{2}$ are the learning factors, $r_{1}$ and $r_{2}$ are random numbers between 0 and 1 , and $\omega$ is the inertia weight coefficient. The range of the solution space is adjusted by adjusting the weight ratio. In $D$-dimensional space, the position of particle $i$ is obtained as

$$
X_{i}(k+1)=X_{i}(k)+V_{i}(k+1) .
$$

3. The iteration stops when the maximum number of iterations is reached or the optimal solution is found by the particle swarm search.

\subsubsection{GPSO}

In the early stage of optimization, the PSO algorithm generally has a faster convergence rate, with all particles close to the optimal position, which leads to the uniformity of the particles, then the convergence rate decreases in the later stage of optimization. For this reason, the crossover and mutation operations in GA are then introduced. By including the characteristics of GA for global optimization, the convergence rate of the particles in the local area is improved, thereby enhancing the search ability of the particles, and realizing the complementarity of the two algorithms. ${ }^{(31)}$ The flowchart of the GPSO algorithm is shown in Fig. 4 and involves the following steps.

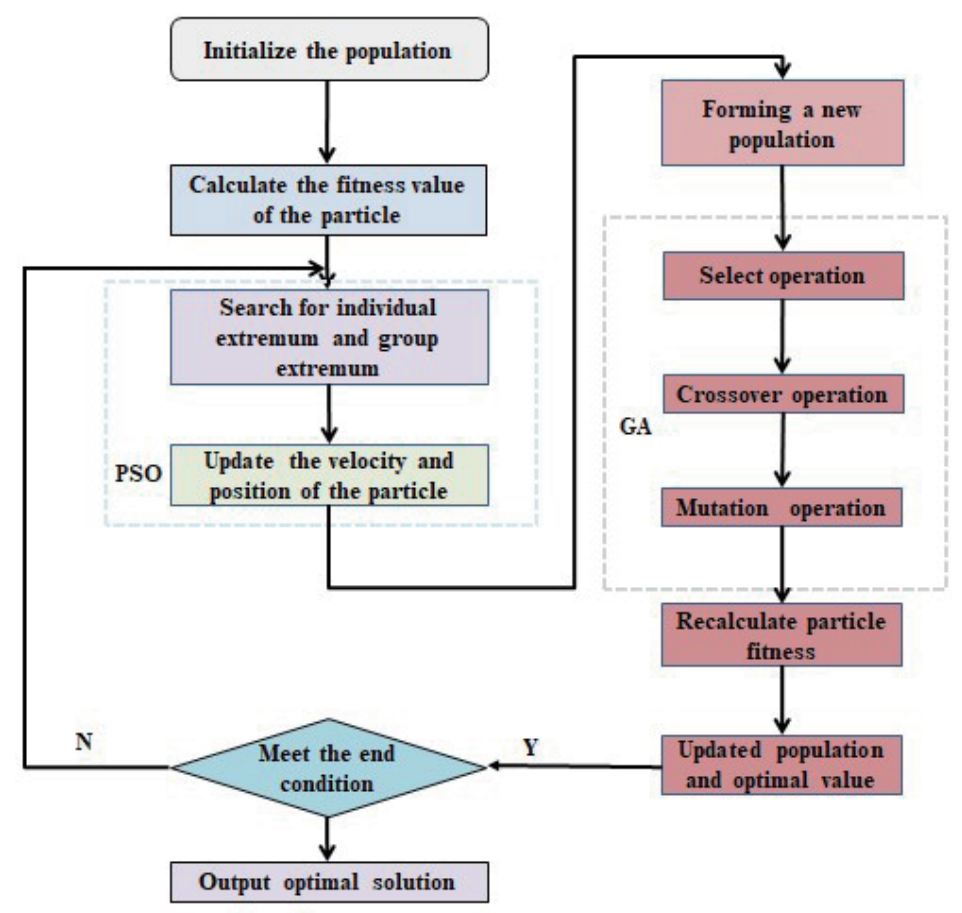

Fig. 4. (Color online) Flowchart of GPSO fusion algorithm. 
1. Randomly initialize the particles in the population and set the corresponding parameters. The number of particles in the population is assumed to be $N$.

2. Calculate the fitness of the individuals in the population, and update the individual extremum and the global extremum in the population according to the fitness. Then obtain the individual optimal value $\left(P_{\text {best }}\right)$ and the global optimal value $\left(G_{\text {best }}\right)$ of the particle swarm.

3. Update the velocity and position of the particles and create a new population.

4. Genetic procedure. Apply the crossover operation to particles $X_{i}$ and $X_{j}$ with probability $P_{c}$. The operations of position and velocity crossover are respectively given by Eqs. (11) and (12), where $b_{1}$ and $b_{2}$ are random numbers in $[0,1]$.

$$
\begin{gathered}
\left\{\begin{array}{l}
X_{i}(k+1)=b_{1} \times X_{i}(k)+\left(1-b_{1}\right) \times X_{j}(k) \\
X_{j}(k+1)=\left(1-b_{1}\right) \times X_{i}(k)+b_{1} \times X_{j}(k)
\end{array}\right. \\
\left\{\begin{array}{l}
V_{i}(k+1)=b_{2} \times V_{i}(k)+\left(1-b_{2}\right) \times V_{j}(k) \\
V_{j}(k+1)=\left(1-b_{2}\right) \times V_{i}(k)+b_{2} \times V_{j}(k)
\end{array}\right.
\end{gathered}
$$

Then, in the position and velocity crossover operations, the new individual is obtained by mutating the particles with probability $P_{m}$ as follows:

$$
\begin{gathered}
X_{i j}=\left\{\begin{array}{l}
X_{i j}+\left(X_{i j}-X_{\max }\right) h(g), r_{1} \geq 0.5, \\
X_{i j}+\left(X_{\min }-X_{i j}\right) h(g), r_{1}<0.5,
\end{array}\right. \\
h(g)=r_{2}\left(1-k / G_{\max }\right) .
\end{gathered}
$$

In Eq. (13), $X_{\max }$ and $X_{\min }$ are the upper and lower bounds of the value for particle $X_{i j}$, respectively, and $G_{\max }$ is the maximum number of evolutionary iterations in Eq. (14).

5. The fitness value of particles in the new population is recalculated, and the optimal solution $\left(P_{\text {best }}, G_{\text {best }}\right)$ of the population is updated according to the fitness value.

6. Determine whether the condition for ending the iteration is satisfied. If it is not satisfied, return to step 2.

The GPSO algorithm takes the PSO algorithm as the main process and integrates the crossover and mutation operations of GA into PSO. This fusion algorithm makes full use of the advantages of GA and PSO to ensure rapid convergence to the global optimal solution.

\subsection{Improvement of BP neural network by GA}

\subsubsection{BP neural network}

The basic idea of a BP neural network is to use the gradient descent method to adjust the weights and threshold of the network so as to minimize the mean square error between the 
actual output value and the expected output value of the network, which is a kind of multilayer feedforward neural network. Figure 5 shows the topology of a three-layer BP neural network comprising an input layer, a hidden layer, and an output layer. Each neuron is fully connected to the nodes in the next layer, while the nodes in the same layer are not connected to each other. ${ }^{(32)}$

The numbers of nodes in the input layer, hidden layer, and output layer of the network are set to $n, l$, and $q$, and the nodes of the input layer, hidden layer, and output layer are denoted as $X_{i}, Y_{i}$, and $Z_{i}$, respectively. The weight of the connection from $X_{i}$ to $Y_{h}$ is $W_{i h}$, and the weight of the connection from $Y_{h}$ to $Z_{j}$ is $W_{h j}$. The threshold of the input layer to the hidden layer is $\alpha_{h}$, the threshold of the hidden layer to the output layer is $b_{j}$, and the excitation function is

$$
g(x)=\frac{1}{1+e^{-x}} .
$$

The output of the hidden layer is

$$
Y_{h}=g\left(\sum_{i=1}^{n} W_{i h} X_{i}+a_{h}\right), h=1,2, \cdots, l,
$$

and the output of the output layer is

$$
Z_{j}=\sum_{h=1}^{l} Y_{h} W_{h j}+b_{j}, j=1,2, \cdots, q .
$$

The error between the expected output value and the actual output value is

$$
E=\frac{1}{2} \sum_{j=1}^{q}\left(O_{j}-Z_{j}\right)^{2}
$$

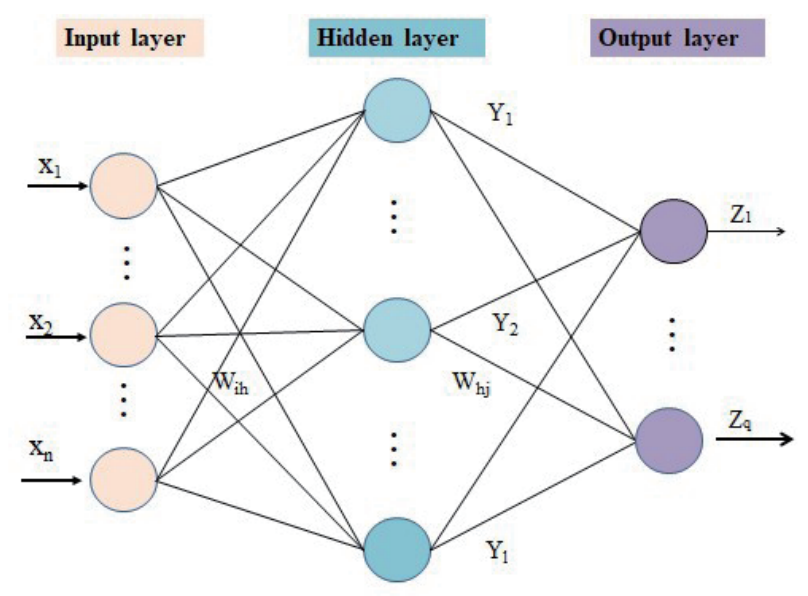

Fig. 5. (Color online) BP neural network structure. 
where $O_{j}$ represents the desired output value and $Z_{j}$ represents the predicted output value obtained through the network.

The error is backpropagated, and the weights and threshold sizes are continuously updated. The training is repeated until the predicted output becomes infinitely close to the actual output.

The weight training procedure of the BP neural network is essentially a complex function optimization problem, and the BP network is very sensitive to the initial weights. In the process of network training, the traditional method of obtaining the weights is to use certain rules and gradually adjust them. In addition, the selection of relevant parameters is often determined according to experience, which causes the convergence rate of the network to be slow and results in an excessively long training time.

\subsubsection{Improvement of BP neural network by GA}

To improve the inherent shortcomings of the BP network, in this study, GA is introduced to optimize the initial weights of the BP network. ${ }^{(33)}$ GA has good global search performance and can obtain the global optimal solution with the maximum probability. Figure 6 shows the implementation process of the GA-BP network, where the preliminary search is completed by GA, which can improve the prediction accuracy and nonlinear mapping ability of BP. The BP neural network is assumed to have three layers, and the steps in BP are as follows:

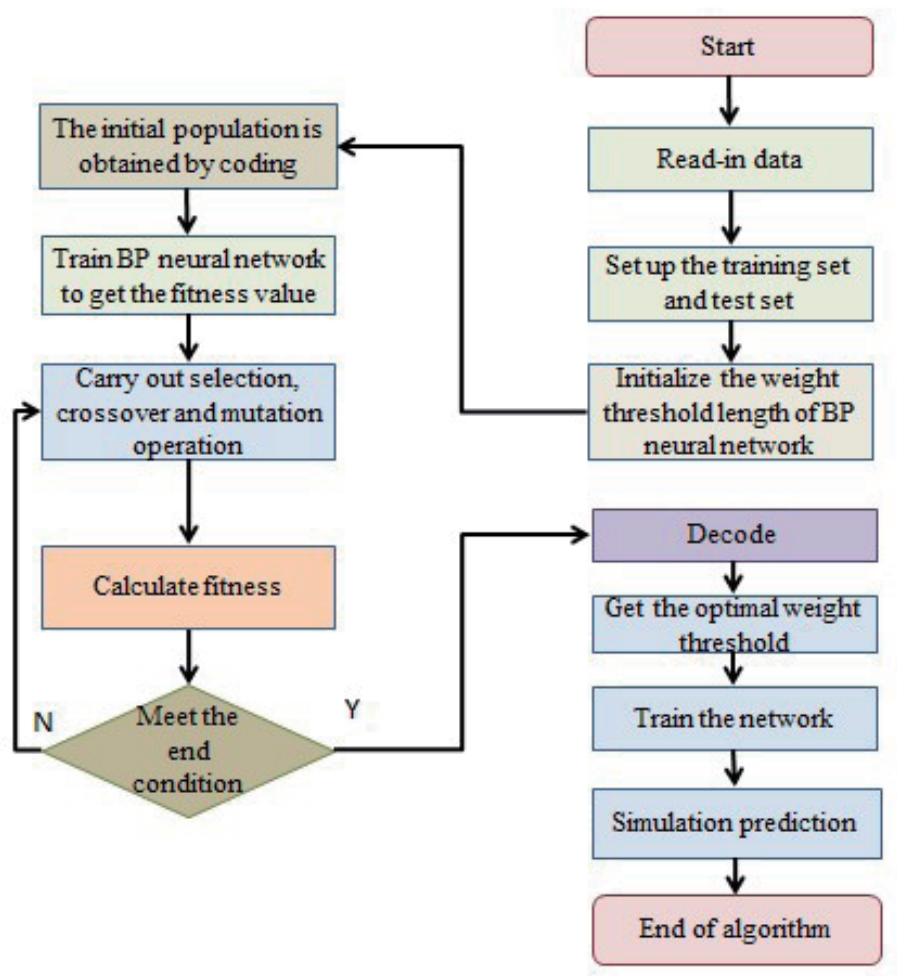

Fig. 6. (Color online) Flowchart of GA-BP. 
1. The weight distribution of a group of neural networks is generated randomly. All weights are connected together in a certain order to form an individual, and then multiple individuals are generated to form a group.

2. Input the training set. Each individual in the population is trained with training samples, and the fitness value of each individual is calculated according to the formula of the learning error, which is

$$
E=\frac{1}{2} \sum_{k=1}^{n} \sum_{j=1}^{q}\left(O_{k j}-Z_{k j}\right)^{2} .
$$

$Z_{k j}$ represents the actual output of the $j$ th neuron node in the output layer of the $k$ th sample and $O_{k j}$ represents the desired output value.

3. The selection operation is sort selection. The individuals are arranged into a sequence according to the fitness value, and the individuals with high fitness enter the next generation as the parents.

4. The arithmetic crossing method (linear crossing) is used in the crossing:

$$
\begin{aligned}
& c_{1}=m \times p_{1}+(1-m) \times p_{2}, \\
& c_{2}=m \times p_{2}+(1-m) \times p_{1},
\end{aligned}
$$

where $m$ is a random number between 0 and $1, p_{1}$ and $p_{2}$ are components of the parent individuals, and $c_{1}$ and $c_{2}$ are the corresponding components of the offspring individuals. In the mutation operation, the relevant weights are set to zero after a neuron is deleted by the mutation operation, and when a neuron is added by the mutation operation, the corresponding initialization weights are generated randomly.

5. Non-uniform variation is used in the mutation operation.

6. Repeat steps 3 to 5 until evolution has been carried out for $m$ generations, and retain the individuals with the best fitness. Use GA to iteratively search for the optimal weights of the BP neural network, then calculate the fitness value of the current population to obtain the optimal individual, which is updated to $P_{\text {best }}$.

7. Obtain the best network weight after decoding $P_{\text {best }}$, then use the BP neural network with the optimal weights to predict the test set.

\subsection{MPE}

MPE can calculate and measure the complexity and uncertainty of a sequence from multiple scales and can accurately reflect the microscale abrupt behavior of the vibration response of a dynamic system with high sensitivity. ${ }^{(34)}$ MPE is improved on the basis of permutation entropy, and the coarse-grained processing of time series is carried out in multiple scales. A time series of length $L,\left\{x_{1}, x_{2}, x_{3}, \ldots, x_{L}\right\}$, is given and coarsened by the following process: 


$$
Z_{j}^{s}=\frac{1}{S} \sum_{i=(j-1) s+1}^{j s} x_{i}, \quad 1 \leq j \leq \frac{L}{s},
$$

where $s$ is the scale factor. When $s=1$, the time series is the original series and the calculated entropy is the permutation entropy. Then, the MPE of the time series is calculated using the permutation entropy algorithm after multiscale decomposition of the sequence.

In terms of analyzing the signal complexity, MPE can consider the possible existence of different time scales in a time series. In this paper, the complexity of IMFs is judged by calculating the mean value of the MPE of different components; the larger the mean value, the more complex the IMF component, and the smaller the mean value, the more regular the IMF component.

\subsection{Proposed model based on GPSO-VMD and improved BP}

The flowchart of the parallel processing model proposed in this paper is shown in Fig. 7, which includes the following steps.

1. The output value of the gyroscope as a function of temperature and the corresponding temperature are obtained through a temperature experiment. Then, the output signal is decomposed by GPSO-VMD, and the optimal VMD parameters $\left(k_{\text {best }}, \alpha_{\text {best }}\right)$ are obtained by GPSO iterative optimization. After decomposition, IMFs are obtained.

2. MPE is used to judge the complexity of each IMF sequence, and these sequences are divided into three parts, noise items, mixed items, and drift items, according to the calculated values. The noise items contain useless noise information, which can be removed directly. The mixed items are smoothed by an FLP filter, and the drift items correspond to the drift of the gyroscope due to changes in temperature. The GA-BP temperature prediction method is adopted to compensate the drift.

3. Finally, the mixed items after FLP filtering and the drift items after temperature compensation are reconstructed to obtain the final output signal.

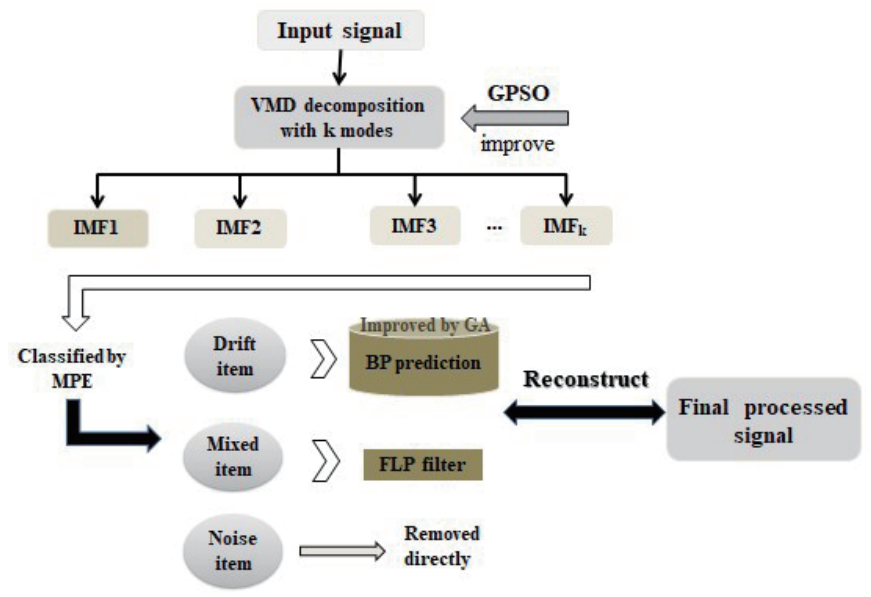

Fig. 7. (Color online) Process of parallel model based on GPSO-VMD and improved BP. 


\section{Experimental Process, Results, and Discussion}

\subsection{Experimental process}

Figure 8 shows the gyroscope and the equipment used in the temperature experiment performed to verify the feasibility of the proposed method. The experimental devices included a temperature-controlled oven, a signal generator (Agilent 33220A), a power supply (Agilent E3631A), a multimeter (Agilent 34401A), an oscilloscope (Agilent DSO7104B), a turntable power supply that can provide $\pm 10 \mathrm{~V}$ DC voltage, and a grounding device. The detection circuit designed in this experiment was placed in three printed circuit boards (PCBs), which were wrapped in a rubber pad and placed in a metal shell. The purpose of this placement was to protect the chip structure of the detection circuit from external impacts while grounding the metal shell to effectively shield the detection circuit from electromagnetic field interference. The three PCBs had different functions. One PCB was connected to the fabric chip structure as an interface for processing weak signals, and the other two were the sensing circuit and driving circuit.

The temperature inside the metal casing of the gyroscope was measured in real time using a thermal resistance. The two output lines of the thermal resistance were connected to a multimeter, and the gyroscope temperature was obtained in real time by adopting the multimeter to obtain the thermal resistance value. Firstly, a tuning-fork MEMS gyroscope was placed in the temperature-controlled oven with a temperature range of -40 to $60{ }^{\circ} \mathrm{C}$. To ensure that the initial temperature of the gyroscope was $60{ }^{\circ} \mathrm{C}$, the experimental equipment was energized for $1 \mathrm{~h}$ at room temperature, and then quickly heated to $60^{\circ} \mathrm{C}$. Then, the temperature of the oven was set to drop by $10{ }^{\circ} \mathrm{C}$ every $1 \mathrm{~h}$ to ensure that the temperature of the gyroscope was consistent with the temperature of the oven. The multimeter collected the output of the gyroscope at a rate of

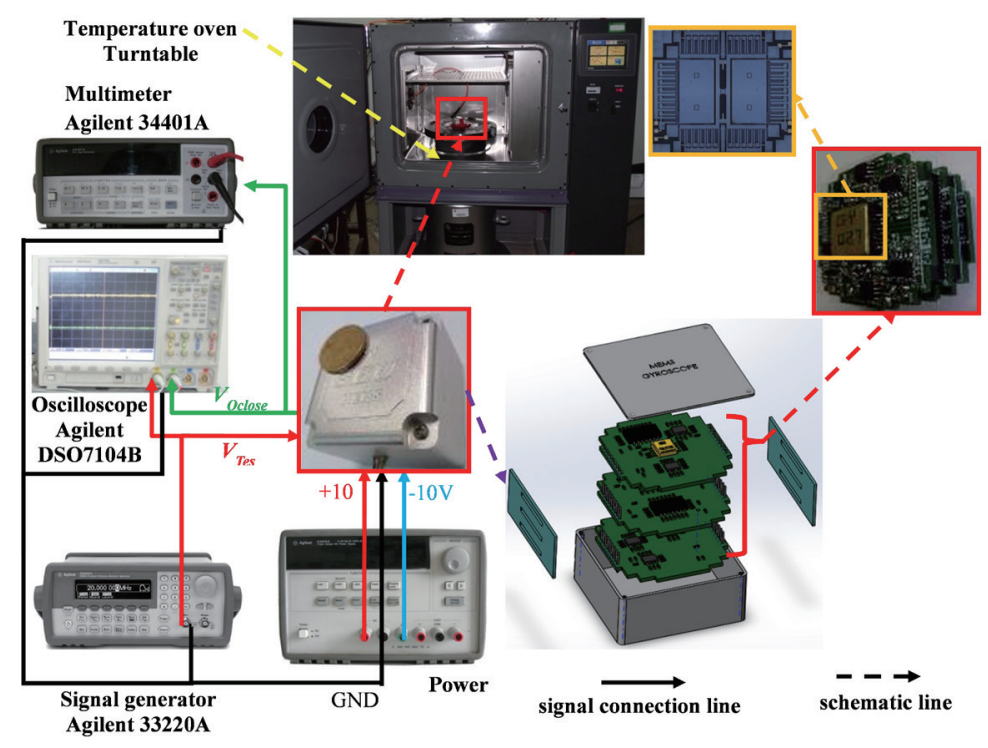

Fig. 8. (Color online) Devices in temperature experiment. 
1000 points/h. After the oven temperature had been maintained at the lowest temperature of $-40{ }^{\circ} \mathrm{C}$ for $1 \mathrm{~h}$, all cooling steps and tests were completed. The measured data are shown in Fig. 9, and the variation curve indicates that the output of the MEMS gyroscope is easily constrained by the temperature. Consequently, the establishment of a temperature compensation model is necessary.

\subsection{Results and discussion}

The experimental results in Fig. 9 indicate that the output of the gyroscope varies greatly with temperature, with a variation of $0.0287 \%$ in the range of -33.67 to $55.67{ }^{\circ} \mathrm{C}$. Meanwhile, there are many burrs in the output signal, indicating a large amount of noise interference. Therefore, it is necessary to remove the noise and compensate the temperature drift of the output signal.

The output signal is decomposed by VMD based on GPSO. The GPSO algorithm is first used to obtain the optimal parameters $\left(k_{\text {best }}, \alpha_{\text {best }}\right)$ of VMD. When VMD is optimized by the GPSO algorithm, the size of the population is set to 50, the range of $k$ is 3 to 11 , and the range of $\alpha$ is 50 to 5000. The optimization results are shown in Fig. 10, and the optimal parameters are $k=11$ and
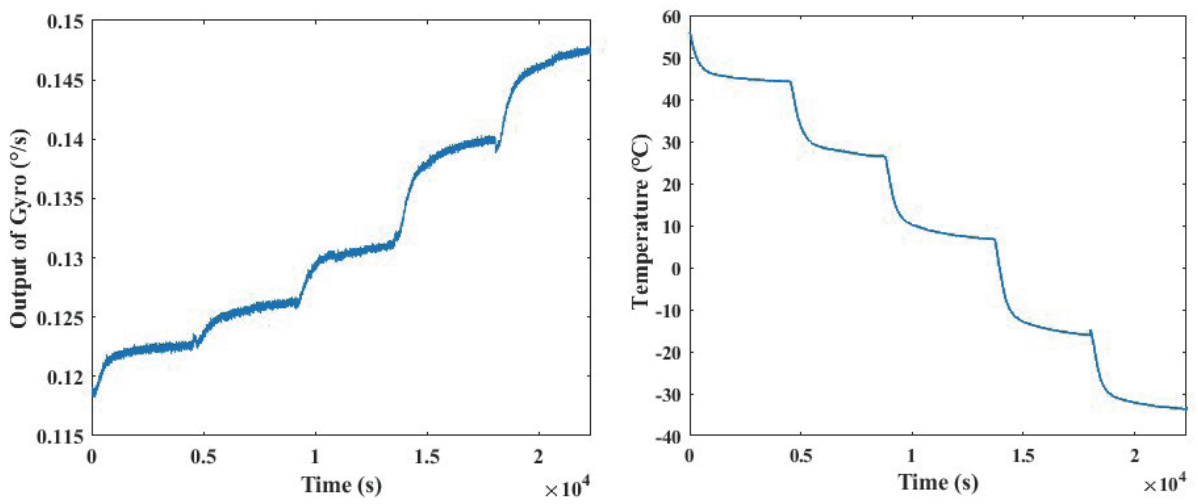

Fig. 9. (Color online) Output signal and temperature change of gyroscope measured by experiment.

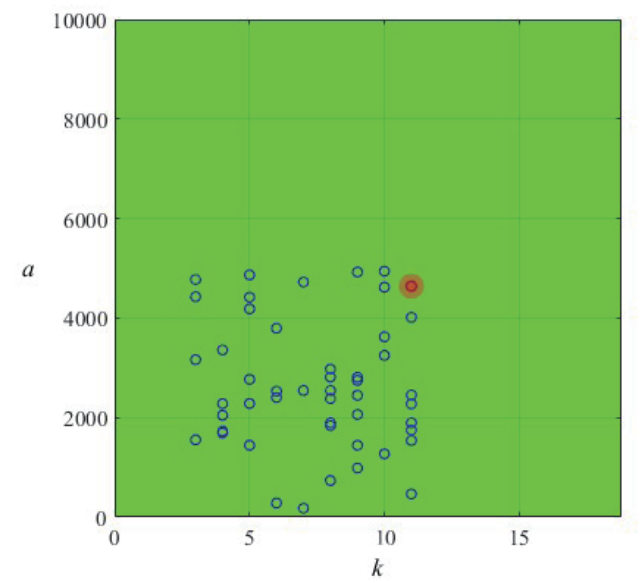

Fig. 10. (Color online) Particle distribution map. 
$\alpha=4641$. Figure 10 also shows the distribution of the optimal particle in all particle swarms. Then, the obtained parameters are used in the VMD to obtain the decomposition results, as shown in Fig. 11. Next, the scale factor is set to 12 and the average value of the MPE of each IMF component is calculated. According to the calculation results, the 11 components are divided into three parts: noise items, mixed items, and drift items (Fig. 12).
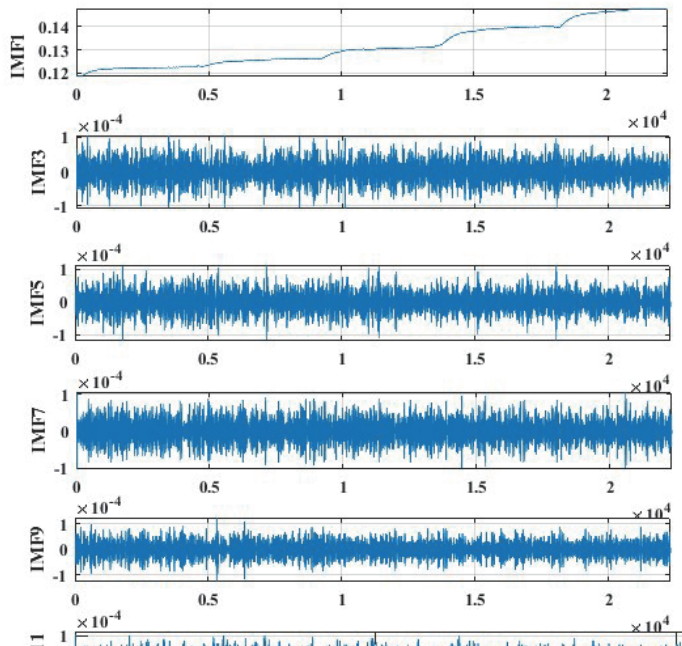

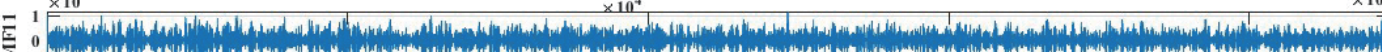

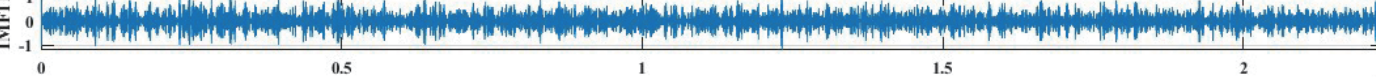

Fig. 11. (Color online) Result of the VMD decomposition of the output signal.

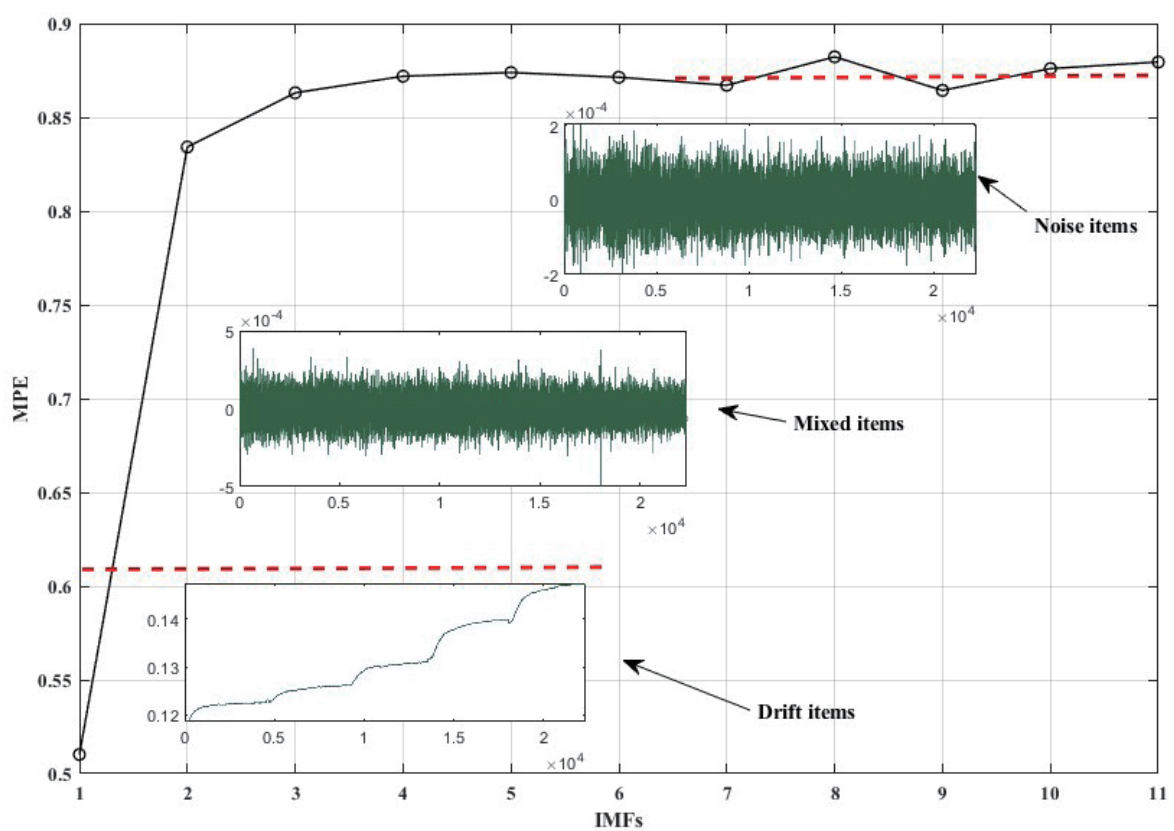

Fig. 12. (Color online) Results of classification using MPE. 
Noise items have a high degree of disorder and contain useless information, which can be removed directly. FLP is used to filter the mixed items. After filtering, the variance of the mixed items is reduced from $1.018 \times 10^{-4}$ to $1.720 \times 10^{-5}$, which shows that the noise interference is effectively removed. Then, the temperature drift term is processed by the BP neural network based on GA, and the final processing results are shown in Fig. 13. Subsequently, the final output signal is obtained by reconstructing the processed drift and mixed items and is shown in Fig. 14.
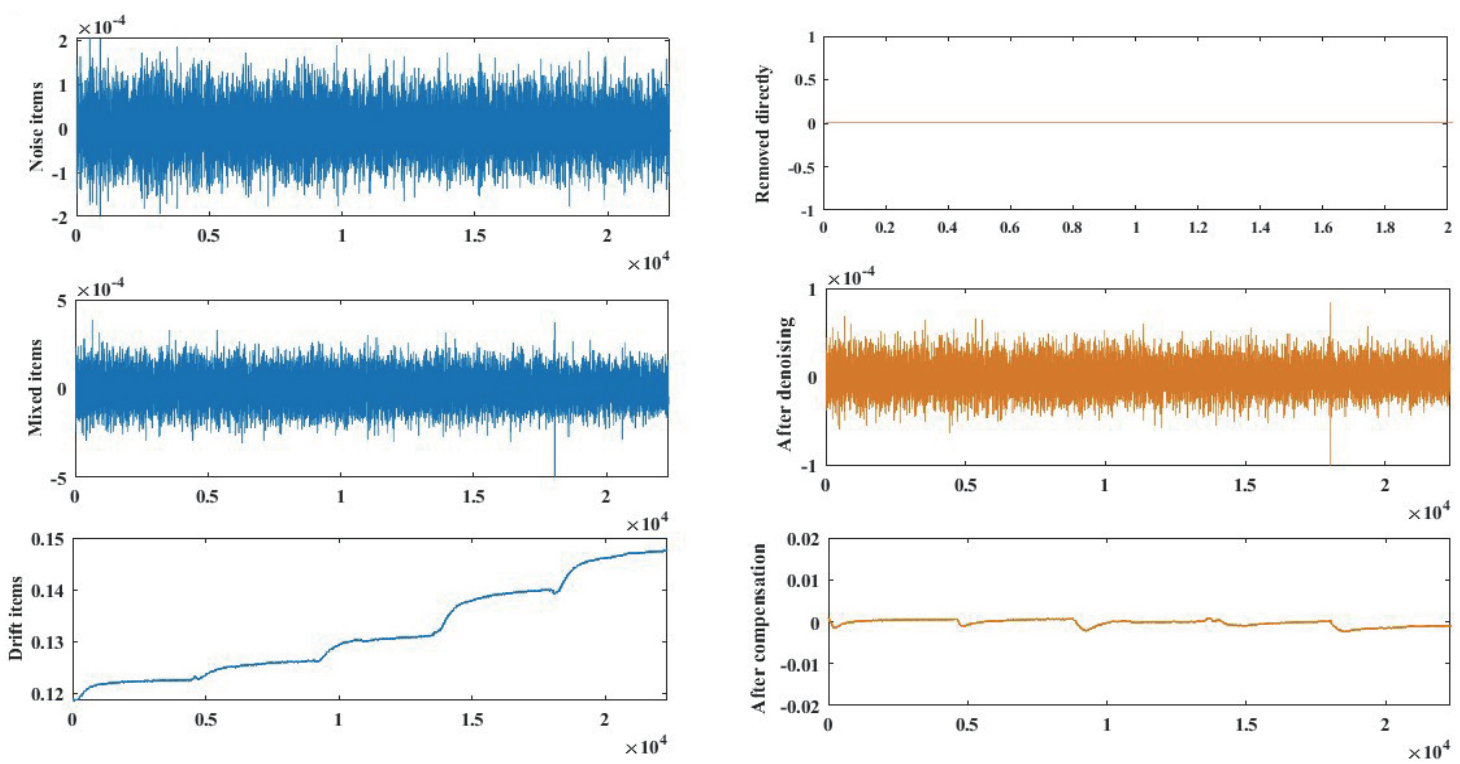

Fig. 13. (Color online) Result of using the model to process the signal.

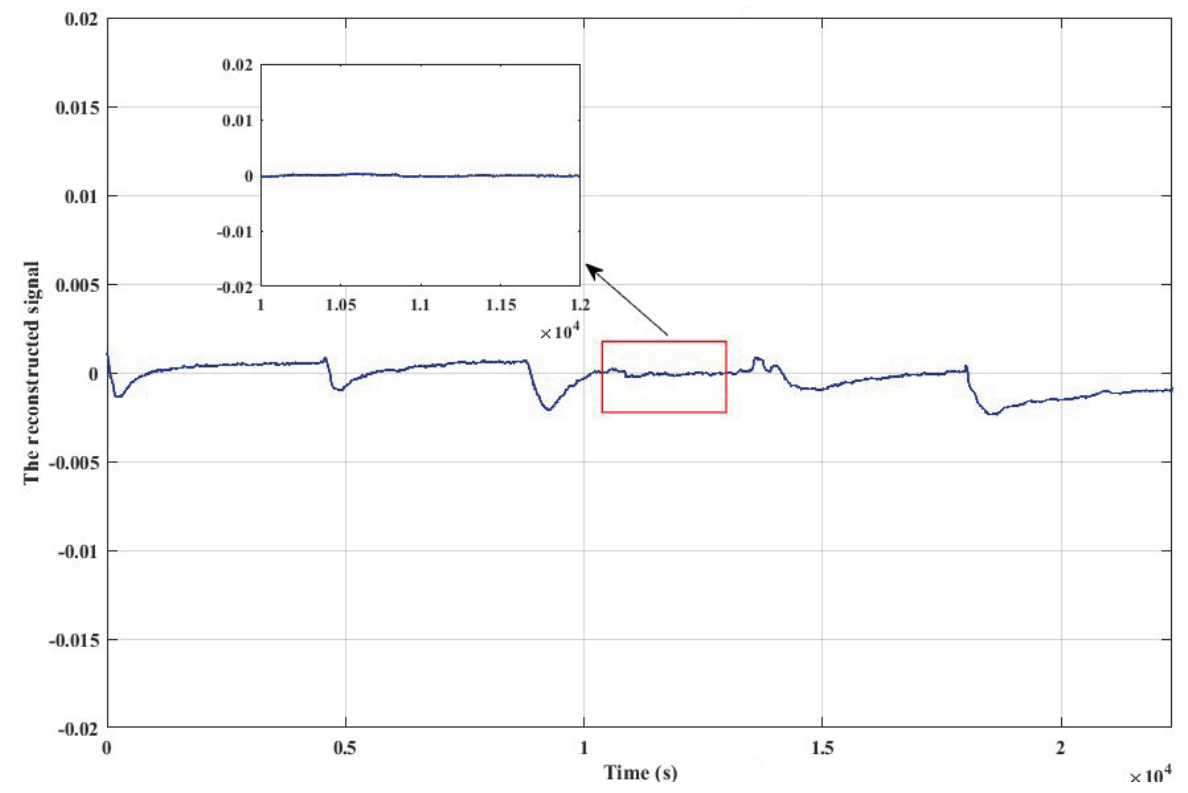

Fig. 14. (Color online) Reconstructed output signal. 


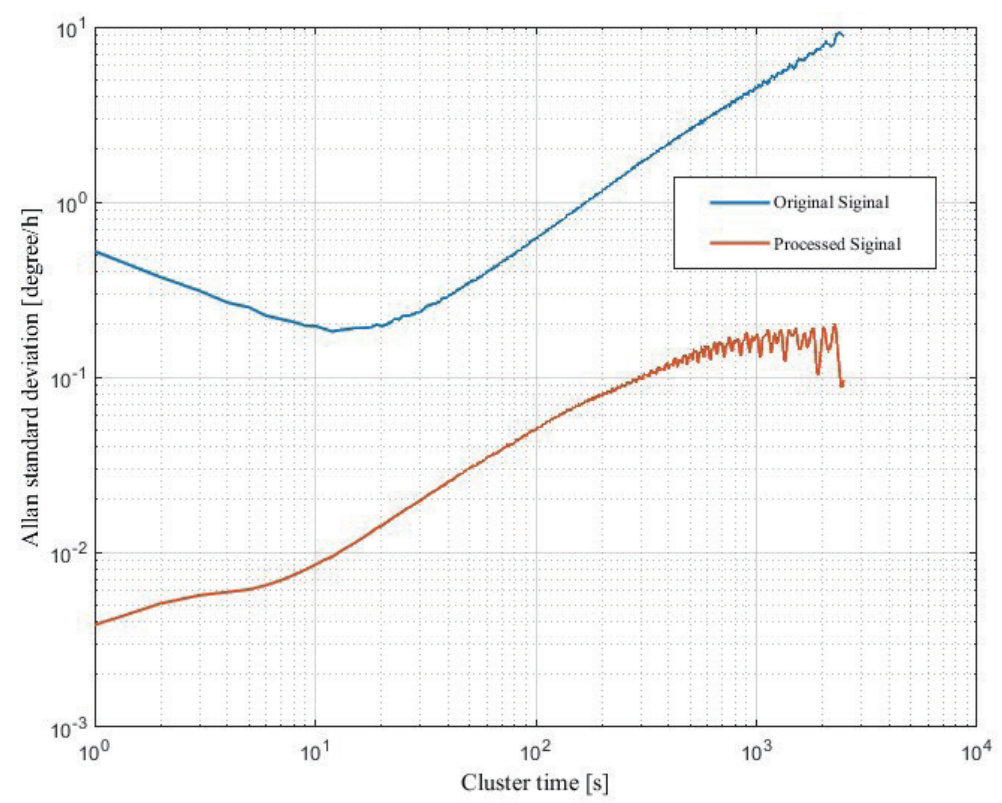

Fig. 15. (Color online) Comparison of Allan variance.

After that, the Allan variance is used to evaluate the performance of the reconstructed signal (Fig. 15). The results show that the processed signal is smoother and has better performance than the unprocessed signal. The angular random walk value was $3.832 \times 10^{-30} / \mathrm{h} / \sqrt{ } \mathrm{Hz}$, compared with $0.0523 \% \mathrm{~h} / \mathrm{JHz}$ for the original signal of the gyroscope, and the bias stability of the processed signal was $0.273 \% \mathrm{~h}$, compared with $31.428 \% \mathrm{~h}$ for the original signal of the gyroscope. These results clearly demonstrate the effectiveness of the parallel processing model of the gyroscope output signal based on GPSO-VMD and GA-BP. However, there are still some defects to be overcome that require further study, such as the excessively long optimization time of the GPSO algorithm.

\section{Conclusions}

We proposed a hybrid parallel processing model based on GPSO-VMD and improved BP to reduce the MEMS gyroscope temperature error. Experimental results showed that the temperature has a strong influence on the output of the gyroscope, and this processing model can effectively extract the features of the gyroscope signal and reduce the noise interference and temperature drift in the output signal. In this model, VMD based on the GPSO algorithm is adopted to decompose the original output signal, where GPSO is introduced to optimize the parameters of VMD to find the optimal solution $\left(k_{\text {best }}, \alpha_{\text {best }}\right)$, and a series of IMFs is obtained after decomposition. Then, MPE is used to divide all the IMFs into three parts: noise items, mixed items, and drift items. Noise items are removed directly, mixed items are filtered with FLP, and drift items are compensated using the GA-BP algorithm. Finally, the filtered mixed items and the compensated drift items are reconstructed to obtain the final output signal. The 
results of numerical analysis show that the processed signal has better performance than the original output signal: the angular random walk value of the compensated signal is reduced by $92.6 \%$ and the bias stability is reduced by $99.1 \%$. Therefore, the proposed parallel processing model can remove the noise interference and greatly eliminate the temperature error in the output signal of the MEMS gyroscope.

\section{Acknowledgments}

This work was supported by the National Natural Science Foundation of China (No. 51705477) and Pre-Research Field Foundation of Equipment Development Department of China (No. 61405170104). It was also supported by the Program for Top Young Academic Leaders of Higher Learning Institutions of Shanxi, the Fund Program for the Scientific Activities of Selected Returned Overseas Professionals in Shanxi Province, Shanxi Province Key Laboratory of Quantum Sensing and Precision Measurement (201905D121001), the Patent Promotion and Implementation Program of Shanxi Province (2019025), Key Research and Development (R\&D) Projects of Shanxi Province (202003D111004), the Aeronautical Science Foundation of China (2019080U0002), and the Shanxi “1331 Project” Key Subjects Construction Fund.

\section{References}

1 D. K. Shaeffer: IEEE Commun. Mag. 51 (2013) 100. https://doi.org/10.1109/MCOM.2013.6495768

2 A. Noureldin, T. B. Karamat, M. D. Eberts, and A. El-Shafie: IEEE Trans. Veh. Technol. 58 (2009) 1077. https://doi.org/10.1109/TVT.2008.926076

3 J. Wendel, O. Meister, C. Schlaile, and G. F. Trommer: Aerosp. Sci. Technol. 10 (2006) 527. https://doi. org/10.1016/j.ast.2006.04.002

4 A. Waegli, J. Skaloud, S. Guerrier, M. E. Parés, and I. Colomina: Meas. Sci. Technol. 21 (2010) 156. https://doi. org/10.1088/0957-0233/21/6/065201

5 S. Sonmezoglu, S. E. Alper, and T. Akin: J. Microelectromech. Syst. 23 (2014) 284. https://doi.org/10.1109/ JMEMS.2014.2299234

6 H. Sheng and T. Zhang: Measurement 59 (2015) 63. https://doi.org/10.1016/j.measurement.2014.09.041

7 G. Zhanshe, C. Fucheng, L. Boyu, C. Le, L. Chao, and S. Ke: Microsyst. Technol. 21 (2015) 2053. https://doi. org/10.1007/s00542-015-2645-X

8 G. Sheng, G. Gao, and B. Zhang: Micromachines 10 (2019) 608. https://doi.org/10.3390/mi10090608

9 J. Georgy, A. Noureldin, M. J. Korenberg, and M. M. Bayoumi: IEEE Trans. Intell. Transp. Syst. 11 (2010) 856. https://doi.org/10.1109/TITS.2010.2052805

10 J. Fang and J. Li: IEEE Trans. Instrum. Meas. 58 (2009) 2923. https://doi.org/10.1109/TIM.2009.2016780

11 I. P. Prikhodko, A. A. Trusov, and A. M. Shkel: Sens. Actuators, A 201 (2013) 517. https://doi.org/10.1016/j. sna.2012.12.024

12 Z Wang, J Zhou, Y Lei, and W Du: Mech. Syst. Sig. Process. 162 (2022) 108018. https://doi.org/10.1016/j. ymssp.2021.108018

13 Z. Wang, N. Yang, and N. Li: Struct. Health Monit. (2021) https://doi.org/10.1177/1475921720986945

14 Z. Wang, W. Zhao, W. Du, N. Li, and J. Wang: Process Saf. Environ. Prot. 149 (2021) 591. https://doi. org/10.1016/j.psep.2021.03.016

15 M. Cui, Y. Huang, W. Wang, and H. Cao: Micromachines 10 (2019) 248. https://doi.org/10.3390/mi10040248

16 Q. Fu, X. P. Di, W. P. Chen, L. Yin, and X. W. Liu: Mod. Phys. Lett. B 31 (2017). https://doi.org/10.1142/ S0217984917500646

17 Q. Zheng, L. Dong, D. H. Lee, and Z. Gao: IEEE Trans. Control Syst. Technol. 17 (2009) 1432. https://doi. org/10.1109/TCST.2008.2008638

18 J. Fei and J. Zhou: IEEE Trans. Syst. Man Cybern. Part B Cybern. 42 (2012) 1599. https://doi.org/10.1109/ TSMCB.2012.2196039

19 H. Cao, Z. Zhang, Y. Zheng, H. Guo, R. Zhao, Y. Shi, and X. Chou: Shock Vib. 2021 (2021) 1. https://doi. org $/ 10.1155 / 2021 / 8855878$ 
20 N. E. Huang, Z. Shen, S. R. Long, M. C. Wu, H. H. Shih, Q. Zheng, N. C. Yen, C. C. Tung, and H. H. Liu: Proc. R. Soc. A-Math. Phy. 454 (1998) 903. https://doi.org/10.1098/rspa.1998.0193

21 G. Cheng, H. Li, X. Hu, X. Chen, and H. Liu: Proc. Inst. Mech. Eng., Part C: J. Mech. Eng. Sci. 231 (2017) 2706. https://doi.org/10.1177/0954406216638885

22 K. Dragomiretskiy and D. Zosso: IEEE Trans. Signal Process. 62 (2014) 531. https://doi.org/10.1109/ TSP.2013.2288675

23 X. B. Wang, Z. X. Yang, and X. A. Yan: IEEE/ASME Trans. Mechatron. 23 (2018) 68. https://doi.org/10.1109/ TMECH.2017.2787686

24 T. Ma, H. Cao, and C. Shen: Electronics 9 (2020) 499. https://doi.org/10.3390/electronics9030499

25 J. K. Shiau, D. M. Ma, C. X. Huang, and M. Y. Chang: Adv. Mater. Res. 255 (2011) 2077. https://doi. org/10.4028/www.scientific.net/AMR.255-260.2077

26 D. Xia, L. Kong, Y. Hu, and P. Ni: Meas. Sci. Technol. 26 (2015) 1. https://doi.org/10.1088/09570233/26/2/025101

27 Y. Li, M. Xu, Y. Wei, and W. Huang: Measurement 77 (2016) 80. https://doi.org/10.1016/j. measurement.2015.08.034

28 H. Cao, R. Cui, W. Liu, T. Ma, Z. Zhang, C. Shen, and Y. Shi: Sens. Rev. 41 (2021) 2. https://doi.org/10.1108/ SR-09-2020-0205

29 Y. Wang, R. Markert, J. Xiang, and W. Zheng: Mech. Syst. Signal Process. 60 (2015) 243. https://doi. org/10.1016/j.ymssp.2015.02.020

30 B. Samanta: Mech. Syst. Sig. Process. 18 (2004) 625. https://doi.org/10.1016/S08883-270(03)000207-

31 A. Gálvez and A. Iglesias: Appl. Soft Comput. 13 (2013) 1491. https://doi.org/10.1016/j.asoc.2012.05.030

32 X. Wang, J. Wang, and M. Privault: J. Intell. Fuzzy Syst. 35 (2018) 4141. https://doi.org/10.3233/JIFS-169735

33 S. Wang, N. Zhang, L. Wu, and Y. Wang: Renewable Energy 94 (2016) 629. https://doi.org/10.1016/j. renene.2016.03.103

34 F. C. Morabito, D. Labate, F. L. Foresta, A. Bramanti, G. Morabito, and I. Palamara: Entropy 14 (2012) 1186. https://doi.org/10.3390/e14071186

\section{About the Authors}

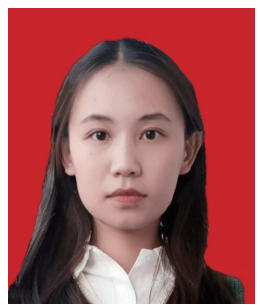

Jingru Wei is currently an undergraduate student at the School of Instrument and Electronics, North University of China, Taiyuan, Shanxi, China. Her research interests lie in MEMS inertial devices. (jingru_www@163.com)

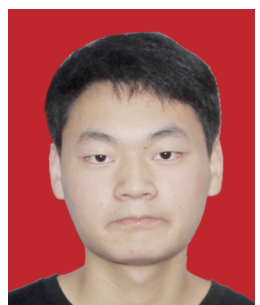

Zekai Zhang is currently an undergraduate student at the School of Instrument and Electronics, North University of China, Taiyuan, Shanxi, China. His research interests lie in MEMS inertial devices.

(zhangzekai_2000@163.com) 


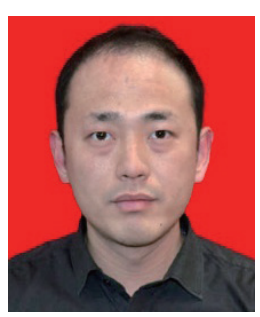

Huiliang Cao received his PhD degree in instrument science and technology from Southeast University, Nanjing, China, in 2014. From 2011 to 2012, he was a research $\mathrm{PhD}$ student in the School of Electrical and Computer Engineering in Georgia Institute of Technology, Atlanta, USA. He is one of the Top Young Academic Leaders of Higher Learning Institutions of Shanxi and Young Academic Leaders of North University of China. He is currently a postgraduate tutor and associate professor of the School of Instrument and Electronics, North University of China, Taiyuan, Shanxi, China. His research interests include MEMS inertial devices. (caohuiliang1986@126.com)

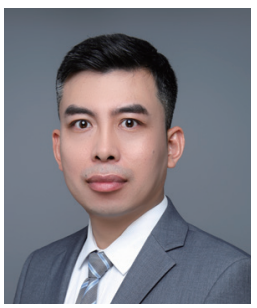

Xiaomin Duan received his $\mathrm{PhD}$ degree in instrument science and technology from North University of China, Taiyuan, China, in 2015. He is currently a postdoctoral researcher at the School of Electronic Science and Engineering, University of Electronic Science and Technology of China, Chengdu, China. His research interests are in the fields of inertial sensors and systems.

(dxm@uestc.edu.cn) 